\title{
An Electroscalar Energy of the Sun: Observation and Research
}

\author{
Oleg Antonovich Zaimidoroga \\ Joint Institute for Nuclear Research, Dubna, Russia \\ Email: Zaimidoroga@jinr.ru
}

Received 3 February 2016; accepted 26 April 2016; published 29 April 2016

Copyright (C) 2016 by author and Scientific Research Publishing Inc.

This work is licensed under the Creative Commons Attribution International License (CC BY).

http://creativecommons.org/licenses/by/4.0/

(c) (i) Open Access

\begin{abstract}
The observation of an electroscalar signal during the eclipse of the Sun by the Moon in 2008 was a starting point for the development and creation of the electroscalar field theory. This observation shows that such radiation has a long wavelength, and is longitudinal and extremely penetrating. The properties of the electroscalar and electromagnetic dynamics of a massive charged particle have been studied. An analogy between the linear theory of elasticity and Maxwell electrodynamics is made. The observed spectrum of radiation clarifying peculiar properties. Real sources of electroscalar radiation are determined. In accordance with the principle of least action, the Lagrangian of the electroscalar field and the field force acting on the particle are defined. The spectral expansion of the electroscalar field allowed us to establish that the field is longitudinal and aligned with the wave vector. At the heart of the electroscalar theory, which is compliant with the experimental data, is the four-dimensional scalar potential that describes radial vibrations of the electroscalar field source. The four-vector Maxwell electromagnetic potential and four-scalar potential neither form a single object in the Minkowski space nor interfere and, as a consequence, prove to be independent and unrelated differential relations. Moreover, a strong correlation between the spatial position of the particle and the field components allows and demonstrates a new degree of freedom in the electrodynamics of charged particles.
\end{abstract}

\section{Keywords}

Four-Scalar Potential of Electroscalar Field, Solar Electroscalar Field, Transport of Coulomb Field

\section{Introduction}

Registration of electroscalar radiation was made with a system of spherical electro-sensors (four copper spheres) placed in a metallic box during an eclipse of the Sun by the Moon which took place on 1 August 2008. The centers of the four spheres were connected to each other with a copper wire [1]. Data taking was done by an 
amplitude-digital analyser. In the proposed theory, physical fields are of two types: vortex electric and magnetic as well as potential electric and three-scalar [2] [3]. The fields of the first type can propagate in vacuum as transverse electromagnetic waves, and the fields of the second type as longitudinal electroscalar waves where the electric field vector vibrates along the propagation direction. It is precisely the longitudinal electroscalar mode that performs relativistic transport of the Coulomb field which is absent in the classical Maxwell theory. The absence of wave transport of the Coulomb field brings about certain obstacles in the canonical quantization of the Maxwell theory [4]-[9] and the violation of the causality principle. In frames of the vector formalism the presence of the longitudinal wave mode leads to the loss of gauge invariance in the theory and to the observability of electrodynamic potentials [10]-[14]. The loss of gauge invariance, in its turn, entails problems related to electric charge conservation. An analogy between the theory of elasticity and electrodynamics is made. The longitudinal electroscalar mode in the proposed theory is a consequence of continuum compressibility, while the Maxwell theory describes the wave dynamics of absolutely incompressible continuum. The electroscalar radiation has a long wavelength, is extremely penetrating, and propagates as a plane wave. In the electroscalar field, the particle energy assumes a negative value to the mechanical energy of the particle. The equation of the electromagnetic field contains displacement current, and the electroscalar field contains displacement charge. The electroscalar field has an ability to convey energy and information in any medium. Constructively, this hypothesis is expressed through the introduction of a four-scalar potential along with the four-vector one. These two four-dimensional fields are not connected with each other by any differential or any other correlations. The above circumstance permits to preserve gauge invariance for transverse fields and avoid difficulties pertaining to the law of electric charge conservation. The experimental proofs of the longitudinal electroscalar mode contain evidence for its presence [15]-[17].

\section{An Analogy with the Theory of Elasticity}

It should be pointed out that the elastic continuum supports propagation of both longitudinal and transverse waves; therefore, an analogy with the elasticity theory will give us a constructive indication of how the equations of generalized electrodynamics should look like. The basic equation of the linear elasticity theory is the Love equation which takes the following form in the absence of any outside forces [18] [19]:

$$
-\ddot{\mathbf{u}}+c_{l}^{2} \nabla \operatorname{divu}-c_{t}^{2} \text { rotrotu }=0,
$$

where the vector $\mathbf{u}$ represents the vector of displacements in an elastic medium; $c_{l}$ and $c_{t}$ are the velocities of propagation of the longitudinal and transverse waves, correspondingly. The displacement vector is the main variable in the linear elasticity theory although not directly observable. Physically observable quantities in this theory are first-order derivatives of $\mathbf{u}$, i.e. $\dot{\mathbf{u}}$ is the velocity of elastic displacements and $\sigma_{i j}=\chi \operatorname{divu}+2 \mu u_{i j}$ is the stress tensor, where $\chi$ and $\mu$ are the elastic constants of the medium and $u_{i j}=1 / 2\left(\partial u_{i} / \partial x_{j}+\partial u_{j} / \partial x_{i}\right)$ is the elastic deformation tensor. With these variables the Love equation looks as follows:

$$
-\frac{\partial^{2} u_{i}}{\partial t^{2}}+\frac{\partial \sigma_{i k}}{\partial x_{k}}=0
$$

but here the wave processes are not arranged into transverse and longitudinal elastic waves. In order to separate the wave processes explicitly, the following designations are introduced [20]:

$$
\mathbf{E}=-\dot{\mathbf{u}}, \quad \mathbf{H}=c_{t} \text { rotu, } \quad \mathrm{W}=c_{l} \text { divu, }
$$

In this case, the Love equation takes the form:

$$
\dot{\mathbf{E}}+c_{l} \nabla \mathrm{W}-c_{t} \mathbf{r o t} \mathbf{H}=0 .
$$

Now, let us apply the rotor and divergence operations to the vector $\mathbf{E}$ :

$$
\begin{aligned}
& \operatorname{rot} E=-\operatorname{rot} \dot{\mathbf{u}}=-\frac{1}{c_{t}} \dot{\mathbf{H}}, \\
& \operatorname{divE}=-\operatorname{div} \dot{\mathbf{u}}=-\frac{1}{c_{l}} \dot{\mathrm{W}} .
\end{aligned}
$$


Thus, the following system of equations can be obtained from the Love equation for the introduced field denotations:

$$
\begin{gathered}
\overrightarrow{\dot{\mathbf{E}}}+c_{l} \nabla \vec{W}-c_{t} \operatorname{rot} \overrightarrow{\mathbf{H}}=0, \\
\frac{1}{c_{t}} \dot{\mathbf{H}}+\operatorname{rot} \mathbf{E}=0, \\
\frac{1}{c_{l}} \dot{\mathbf{W}}+\operatorname{div} \mathbf{E}=0, \\
\operatorname{div} \mathbf{H}=0,
\end{gathered}
$$

which, in the event of $\mathrm{W}=c_{l} \operatorname{divu}=0$ corresponding to the incompressible elastic continuum, coincides with the system of Maxwell equations in vacuum:

$$
\begin{aligned}
\dot{\mathbf{E}}-c_{t} \operatorname{rot} \mathbf{H} & =0, \\
\frac{1}{c_{t}} \dot{\mathbf{H}}+\operatorname{rot} \mathbf{E} & =0 .
\end{aligned}
$$

If the continuous medium does not support rotating motion, for which rotu $=0$ (e.g. liquid or gas), then compressible system takes the form:

$$
\begin{aligned}
\dot{\mathbf{E}}+c_{l} \nabla \mathrm{W} & =0, \\
\frac{1}{c_{l}} \dot{\mathrm{W}}+\operatorname{div} \mathbf{E} & =0 .
\end{aligned}
$$

This system describes the propagation of longitudinal waves in a continuous medium. Because the transverse and longitudinal waves propagate in the elastic continuum with different velocities, $c_{t}$ for the transverse and $c_{l}$ for the longitudinal waves, the fields $\mathbf{E}_{\perp}$ and $\mathbf{E}_{\|}$can be deemed as independent. Eventually, the following field definitions are introduced:

$$
\begin{aligned}
& \mathbf{E}_{\perp}=-\nabla \varphi-\frac{1}{c} \frac{\partial \mathbf{A}}{\partial t}, \quad \mathbf{H}=\mathbf{r o t} \mathbf{A} \\
& \mathbf{E}_{\|}=\nabla \lambda, \quad \mathrm{W}=-\frac{1}{c} \frac{\partial \lambda}{\partial t},
\end{aligned}
$$

where $\varphi$ and $\mathbf{A}$ are the time and space parts of the electromagnetic four-potential. It should be noted that by their definitions the fields $\mathbf{E}_{\|}$and $\mathrm{W}$ are the components of a four-vector in the Minkowski space-time. Definitions (8) permit to obtain from Equations (5) and (7) two systems of equations, one for the fields $\mathbf{E}_{\perp}$ and H

$$
\begin{aligned}
& -\frac{1}{c} \frac{\partial \mathbf{E}_{\perp}}{\partial t}+\operatorname{rot} \mathbf{H}=0, \\
& \frac{1}{c} \frac{\partial \mathbf{H}}{\partial t}+\mathbf{r o t} \mathbf{E}_{\perp}=0, \\
& \operatorname{div} \mathbf{H}=0,
\end{aligned}
$$

and another for the fields $\mathbf{E}_{\|}$and $W$ :

$$
\begin{aligned}
& \frac{1}{c} \frac{\partial \mathbf{E}_{\|}}{\partial t}+\nabla \mathrm{W}=0, \\
& \frac{1}{c} \frac{\partial \mathrm{W}}{\partial t}+\operatorname{div}_{\|}=0, \\
& \operatorname{rot}_{\|}=0 .
\end{aligned}
$$


In that event, system (9) describes the propagation of strictly transverse waves, and system (10), of longitudinal waves. Now, let us consider the solution of this system in the form of plane waves $\mathbf{E}_{\|}=\mathbf{E}_{0} \exp [i(\omega t+\mathbf{k r})]$, $\mathrm{W}=\mathrm{W}_{0} \exp [i(\omega t+\mathbf{k r})]$, where $\mathbf{E}_{0}$ and $\mathrm{W}_{0}$ are the amplitudes, and $\mathbf{k}$ is the wave vector which determines the direction of propagation of a wave with the frequency $\omega$. Then, from (10) the following is obtained:

$$
\frac{\omega}{c} \mathbf{E}_{0}+\mathbf{k W} \mathrm{W}_{0}=0, \quad \frac{\omega}{c} \mathrm{~W}_{0}+\left(\mathbf{k} \mathbf{E}_{0}\right)=0, \quad \mathbf{k} \times \mathbf{E}_{0}=0,
$$

i.e. the vector of the electric field vibrates along the direction of wave propagation. The dispersion relation for this wave has the form $\omega=k c$.

\section{Sources of the Electroscalar Wavefield}

The electroscalar field is produced by the time-dependent, inhomogeneous density of an electric charge or part of the transfer current density with non-zero divergence. This source can be well illustrated by the radial oscillations of electron gas in a spherically symmetric metallic particle.

In Figure 1, the arrows correspond to the radial currents in the electron gas (electron convection current); the left part reflects the process of expansion of the electron gas, while the right part demonstrates compression. Due to the spherical symmetry, the magnetic field of the fluctuating radial transfer current equals zero; therefore, the electromagnetic flux is absent. Thus, the radiation losses in this system may be realized at the expense of the longitudinal electroscalar wave radiation. Let us consider the radial motions in the electron gas which are excited by light in a metallic nanoparticle. Here radial motions are such macroscopic motions of the electron gas for which divergence of the transfer current is non-zero and the electron density determines the drift velocity. An example of radial motions in conductive media are plasmons which, from the macroscopic viewpoint, present a collective wave process, i.e. compression and expansion waves in the electron gas which are accompanied by the irrotational vector potential A $(H=\operatorname{rot} A=0)$. The electroscalar waves can be also produced as electrically charged particles slow down in matter (braking electroscalar radiation). The scheme of such a source is given in the following Figure 2.
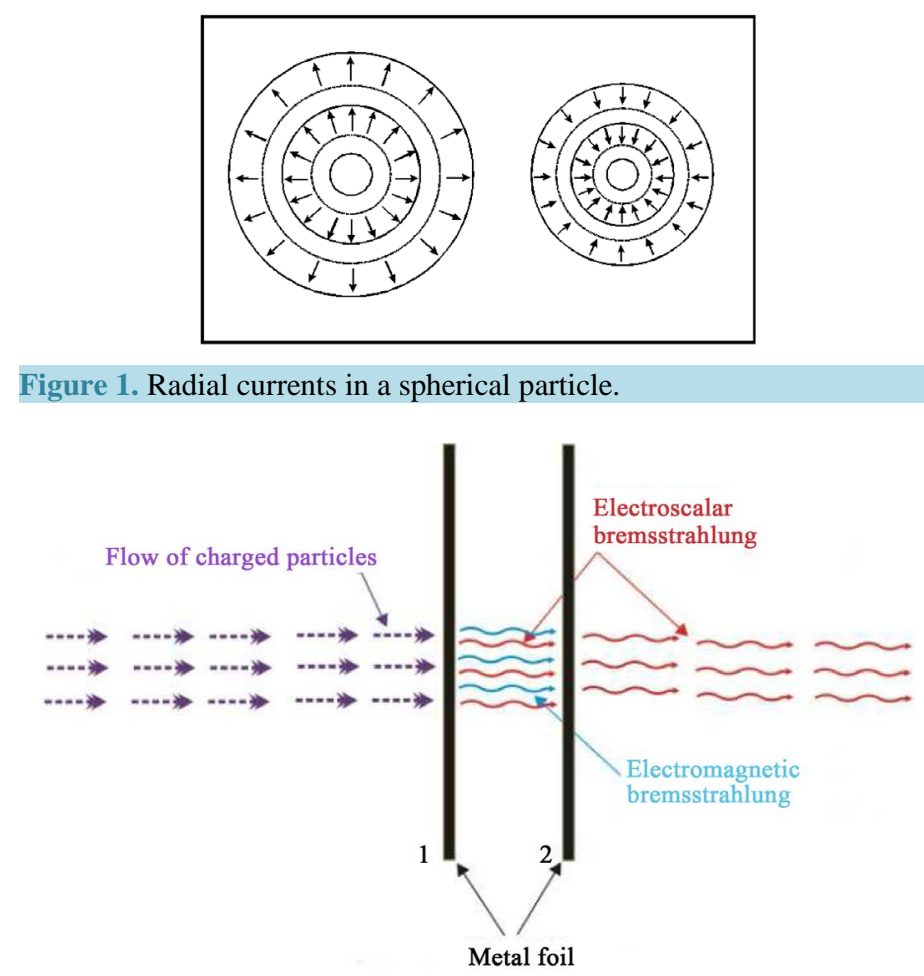

Figure 2. A source of braking electroscalar radiation. 
In the case we consider, the charged particles of a pulsed beam (a high-current electron beam or focused ion beam) stopping in metallic foil 1 serve as a source of braking electromagnetic and electroscalar radiation. The electromagnetic radiation is absorbed by foil 2 (the thickness of foil 2 must exceed the respective thickness of the skin-layer for the braking electromagnetic radiation), and the electroscalar radiation can be registered, due to its greater penetrating power, behind foil 2 in which partial absorption of the electroscalar waves will certainly occur.

\section{The Scalar Field In Presence of Charges and Currents}

Let us consider the interaction of fields with charges and currents supposing that the time component of the four-potential $A^{0}=\varphi$ equals zero, and the three-vector $\mathbf{A}$ satisfies the Coulomb condition $\operatorname{div} \mathbf{A}=0$. Then, the Maxwell electric field becomes a vortex one:

$$
\mathbf{E}_{\perp i}=-\frac{1}{c} \frac{\partial \mathbf{A}_{\perp i}}{\partial t} \quad i=0,1,2,3 .
$$

The vacuum equations for the potentials are:

$$
\begin{aligned}
& -\frac{1}{c^{2}} \frac{\partial^{2} \mathbf{A}}{\partial t^{2}}+\Delta \mathbf{A}=0, \\
& -\frac{1}{c^{2}} \frac{\partial^{2} \lambda}{\partial t^{2}}+\Delta \lambda=0 .
\end{aligned}
$$

In order to derive potential equations for the case with presence of charges, the Lagrangian formalism will be used. Three-dimensional Lagrangians corresponding to (11) have the form:

$$
\begin{aligned}
& L_{E H}=\frac{\mathbf{E}_{\perp}^{2}-\mathbf{H}^{2}}{8 \pi}=\frac{1}{8 \pi}\left(\frac{1}{c} \frac{\partial \mathbf{A}}{\partial t}\right)^{2}-\frac{1}{8 \pi}(\operatorname{rot} \mathbf{A})^{2}, \\
& L_{E W}=\frac{W^{2}-\mathbf{E}_{\|}^{2}}{8 \pi}=\frac{1}{8 \pi}\left(\frac{1}{c} \frac{\partial \lambda}{\partial t}\right)^{2}-\frac{1}{8 \pi}(\nabla \lambda)^{2} .
\end{aligned}
$$

If we introduce the interactions $(\mathbf{A j}) / c$ for $L_{E H}$ and $\rho \lambda$ for $L_{E W}$ :

$$
\begin{gathered}
L_{E H}=\frac{\mathbf{E}_{\perp}^{2}-\mathbf{H}^{2}}{8 \pi}=\frac{1}{8 \pi}\left(\frac{1}{c} \frac{\partial \mathbf{A}}{\partial t}\right)^{2}-\frac{1}{8 \pi}(\operatorname{rot} \mathbf{A})^{2}+\frac{1}{c} \mathbf{A} \mathbf{j}, \\
L_{E W}=\frac{\mathrm{W}^{2}-\mathbf{E}_{\|}^{2}}{8 \pi}=\frac{1}{8 \pi}\left(\frac{1}{c} \frac{\partial \lambda}{\partial t}\right)^{2}-\frac{1}{8 \pi}(\nabla \lambda)^{2}+\rho \lambda,
\end{gathered}
$$

then system (11) takes the form:

$$
\begin{aligned}
& -\frac{1}{c^{2}} \frac{\partial^{2} \mathbf{A}}{\partial t^{2}}+\Delta \mathbf{A}=-\frac{4 \pi}{c} \mathbf{j}, \\
& -\frac{1}{c^{2}} \frac{\partial^{2} \lambda}{\partial t^{2}}+\Delta \lambda=-4 \pi \rho,
\end{aligned}
$$

where $\rho$ and $\mathbf{j}$ are the charge and current densities. Since the vector potential $\mathbf{A}$ satisfies the Coulomb condition, only the vortex part of the transport current will be included, i.e. $\operatorname{div} \mathbf{j}=0$. Thus, the continuity equation

$$
\frac{\partial \rho}{\partial t}+\operatorname{divj}=0
$$

does not impose any constraints on the potentials $\mathbf{A}$ and $\lambda$. Substitution in (9) of the definitions of the fields (8) gives: 


$$
\begin{aligned}
& -\frac{1}{c} \frac{\partial \mathbf{E}_{\perp}}{\partial t}+\operatorname{rot} \mathbf{H}=\frac{4 \pi}{c} \mathbf{j}, \\
& \frac{1}{c} \frac{\partial \mathbf{H}}{\partial t}+\operatorname{rot} \mathbf{E}_{\perp}=0, \\
& \operatorname{div} \mathbf{H}=0,
\end{aligned}
$$

and

$$
\begin{aligned}
& \frac{1}{c} \frac{\partial \mathbf{E}_{\|}}{\partial t}+\nabla \mathrm{W}=0, \\
& \frac{1}{c} \frac{\partial \mathrm{W}}{\partial t}+\operatorname{div} \mathbf{E}_{\|}=-4 \pi \rho, \\
& \operatorname{rot}_{\|}=0 .
\end{aligned}
$$

It is seen from the above equation that the electrostatics equation, $\operatorname{div} \mathbf{E}_{\|}=-4 \pi \rho$, follows from system (17) and the magnetostatics equation, $\operatorname{rot} \mathbf{H}=4 \pi \mathbf{j} / c$, from (16). These two systems of Equations (16) and (17) can be rewritten in the form of wave equations for the fields:

$$
\begin{array}{ll}
-\frac{1}{c^{2}} \frac{\partial^{2} \mathbf{E}_{\perp}}{\partial t^{2}}+\Delta \mathbf{E}_{\perp}=\frac{4 \pi}{c^{2}} \frac{\partial \mathbf{j}}{\partial t}, & \operatorname{div} \mathbf{E}_{\perp}=0, \\
-\frac{1}{c^{2}} \frac{\partial^{2} \mathbf{H}}{\partial t^{2}}+\Delta \mathbf{H}=-\frac{4 \pi}{c^{2}} \text { rotj, } & \operatorname{div} \mathbf{H}_{\perp}=0 ;
\end{array}
$$

and

$$
\begin{gathered}
-\frac{1}{c^{2}} \frac{\partial^{2} \mathbf{E}_{\|}}{\partial t^{2}}+\Delta \mathbf{E}_{\|}=-4 \pi \nabla \rho, \quad \operatorname{rot}_{\|}=0, \\
-\frac{1}{c^{2}} \frac{\partial^{2} \mathrm{~W}}{\partial t^{2}}+\Delta \mathrm{W}=\frac{4 \pi}{c} \frac{\partial \rho}{\partial t} .
\end{gathered}
$$

The identity $\Delta \equiv-$ rotrot $+\nabla \operatorname{div}$ was used while deriving these equations. It is seen from these equations that the time-varying current with a rotor different from zero is the source of transverse waves, while the timedependent non-uniform charge density serves as the source of longitudinal waves. Here, taking into account continuity Equation (15) the later Equation (19) can be presented in the form:

$$
-\frac{1}{c^{2}} \frac{\partial^{2} \mathrm{~W}}{\partial t^{2}}+\Delta \mathrm{W}=-\frac{4 \pi}{c} \operatorname{divj}
$$

It is obvious from (17) and (19) that longitudinal electroscalar waves respond to the transport of the Coulomb field.

The energy conservation laws for (16) and (17) have the form:

$$
\begin{gathered}
\frac{\partial}{\partial t} \int \epsilon_{E H} \mathrm{~d} V+\oint \mathbf{s}_{E H} \mathrm{~d} \sigma=-\int\left(\mathbf{j} \mathbf{E}_{\perp}\right) \mathrm{d} V, \\
\frac{\partial}{\partial t} \int \epsilon_{E W} \mathrm{~d} V+\oint \mathbf{s}_{E W} \mathrm{~d} \sigma=-c \int(\rho \mathrm{W}) \mathrm{d} V,
\end{gathered}
$$

where $\epsilon_{E W}$ and $\mathbf{s}_{E W}$ are the energy density and the flow vector of the electroscalar field, respectively. The electromagnetic energy density $\epsilon_{E H}$ and the flux vector $\mathbf{s}_{E H}$ have the form:

$$
\epsilon_{E W}=\frac{\mathbf{E}_{\|}^{2}+\mathrm{W}^{2}}{8 \pi}, \quad \mathbf{s}_{E W}=\frac{c}{4 \pi} \mathbf{E}_{\|} \mathrm{W},
$$




$$
\epsilon_{E H}=\frac{\mathbf{E}_{\perp}^{2}+\mathbf{H}}{8 \pi}, \quad \mathbf{s}_{E H}=\frac{c}{4 \pi}\left[\mathbf{E}_{\perp} \times \mathbf{H}\right] .
$$

The quantities on the right sides of 21 and (22) determine the energy dissipation of the fields per unit time as they interact with charges and currents. Of particular interest is the integrand $\rho \mathrm{W}$ in the right side of (22). Using the definition $\mathrm{W}=-\partial \lambda / \partial(c t)$ and continuity Equation (15) this expression can be brought to the following form:

$$
\rho \mathrm{W}=-\rho \frac{1}{c} \frac{\partial \lambda}{\partial t}=-\frac{\partial}{\partial t}\left(\frac{1}{c} \lambda \rho\right)+\frac{1}{c} \lambda \frac{\partial \rho}{\partial t}=-\frac{\partial}{\partial t}\left(\frac{1}{c} \lambda \rho\right)-\operatorname{div}\left(\frac{1}{c} \lambda \mathbf{j}\right)+\frac{1}{c} \mathbf{j} \nabla \lambda .
$$

Owing to the field definition $\mathbf{E}_{\|}=\nabla \lambda$, (22) can be represented as:

$$
\frac{\partial}{\partial t} \int\left(\epsilon_{E W}-\rho \lambda\right) \mathrm{d} V+\oint\left(\mathbf{s}_{E W}-\lambda \mathbf{j}\right) \mathrm{d} \sigma=-\int\left(\mathbf{j E}_{\|}\right) \mathrm{d} V .
$$

The first integral is the total energy of the system of fields $\mathbf{E}_{\|}$and $W$ interacting with charges. The summand $\lambda \mathbf{j}$ is added to the surface integral and can be interpreted as energy transfer at the expense of the motion of charges. Now, taking into account that $\mathbf{j}=\rho \mathbf{v}$, one obtains $\lambda \mathbf{j}=(\rho \lambda) \mathbf{v}$. In other words, the total flux consists of two components. The first one is responsible for the energy transfer by means of electroscalar radiation and the other, for the (mechanical) reemission of charges from the area limited by the integration surface.

\section{The Continuity Equation of the Electroscalar Field}

The equation of continuity determines the variation in the quantity of charges in a volume per unit time both leaving and entering this volume through the surface that limits such volume during the propagation of the electroscalar field. From the equation for the electroscalar field it follows that:

$$
\operatorname{div} \mathbf{E}_{\|}=-4 \pi \rho_{\|}-\frac{1}{c} \frac{\partial \mathrm{W}}{\partial t} .
$$

The electroscalar field is determined both by charged particles and variation of the scalar charge in time:

$$
\varrho_{\|}=\rho_{\|}+\frac{1}{4 \pi} \frac{1}{c} \rho_{0},
$$

where $\frac{\partial \mathrm{W}}{\partial t}=\rho_{0}$ is the charge displacement owing to:

$$
\operatorname{div} \mathbf{E}_{\|}=-4 \pi \varrho_{\|} .
$$

It should be noted that the displacement of scalar charge has a wave nature and, therefore, this term should be averaged in time. Thus, the total number of charges in a volume which pass through the surface $\mathrm{S}$ limiting such volume is $\varrho_{\|} v \mathrm{~d} S$ :

$$
\frac{\partial}{\partial t} \int_{a}^{b} \varrho_{\|} \mathrm{d} V=-\int_{S}\left(4 \pi \rho_{\|} v+\rho_{0} v\right) \mathrm{d} S
$$

Further, using the generalized Gauss theorem the continuity equation of the electroscalar field acquires the form:

$$
\frac{\partial}{\partial t} \int_{a}^{b} \varrho_{\|} \mathrm{d} V=-\int_{a}^{b}(4 \pi \operatorname{divj}+\operatorname{divy}) \mathrm{d} V
$$

where the second term is the wave component at the expense of charge displacement. Finally:

$$
\frac{\partial \varrho_{\|}}{\partial t}=-4 \pi \operatorname{divj}-\operatorname{divy}
$$


Note that in the absence of charged currents in vacuum the scalar term (of charge displacement) does not vanish and allows the electroscalar waves to travel in vacuum due to the reproduction of the charges and their fields during the propagation.

\section{The Superposition Principle of the Electroscalar Field}

The longitudinality of the wave imposes certain requirements on the action of the field, particularly, on such a property of the field as the superposition principle. The equations for the electromagnetic field (Maxwell equations) contain only the equations for the full charge conservation (equations of continuity) but not the equations of motion for the field producing charges. In the case of the electromagnetic field, the distribution and motion of charges can be specified arbitrarily provided that the full charge is conserved. The character of the charges' distribution is determined then by solving the Maxwell equations using the field produced by the charges. As experience shows, the electromagnetic field obeys this principle which implies that if a charge produces a field, and another charge produces another field, then the total field is a result of superposition of these fields. Such a superposition principle will be referred to as the method of transverse summation of fields. The equations of motion of the electroscalar field contain not only the equations of continuity and full charge conservation, which cannot be determined arbitrarily. But the field itself and the charges' motion must be determined concurrently with the field of the produced charges' field. The principle of superposition of the electroscalar field is longitudinal as summation of the fields' charges occurs only for those located along the line between the charges. This means that the strengths of the resultant electroscalar fields at each point are equal to a sum of the strengths of all the longitudinal fields at this point. Any solution of equations for these fields is a field that can be realized in nature and, consequently, must obey the electroscalar field equations. In the electroscalar field the distribution of charges and their motion must be defined by solving field equations with given initial conditions for the longitudinal superposition of charges. Thus, while radiation is propagating, both the charges and their fields are reproduced in compliance with the electroscalar field laws, and the field potentials and strengths are enhanced due to the linear law of summation of the charges' fields. We will now consider how an electroscalar wave propagates between two charges (for example, of electrons). In this case, the field's electrical component is responsible for the local deviation of the electron density from the equilibrium one, whereas compression of the electric vacuum takes place under the action of the field's scalar component. The passage of the electroscalar radiation through two charges leads to a standing wave with a knot at the charge. Further, the wave passes in this manner all the charges located along the line of its propagation. The increased amplitude of the charges' density and field strength gives rise to a chain of compression and expansion waves up to the arrival at the target. The photo displays the structure of the compression and expansion zones in an electroscalar wave in the atmosphere [21] presents on Figure 3.

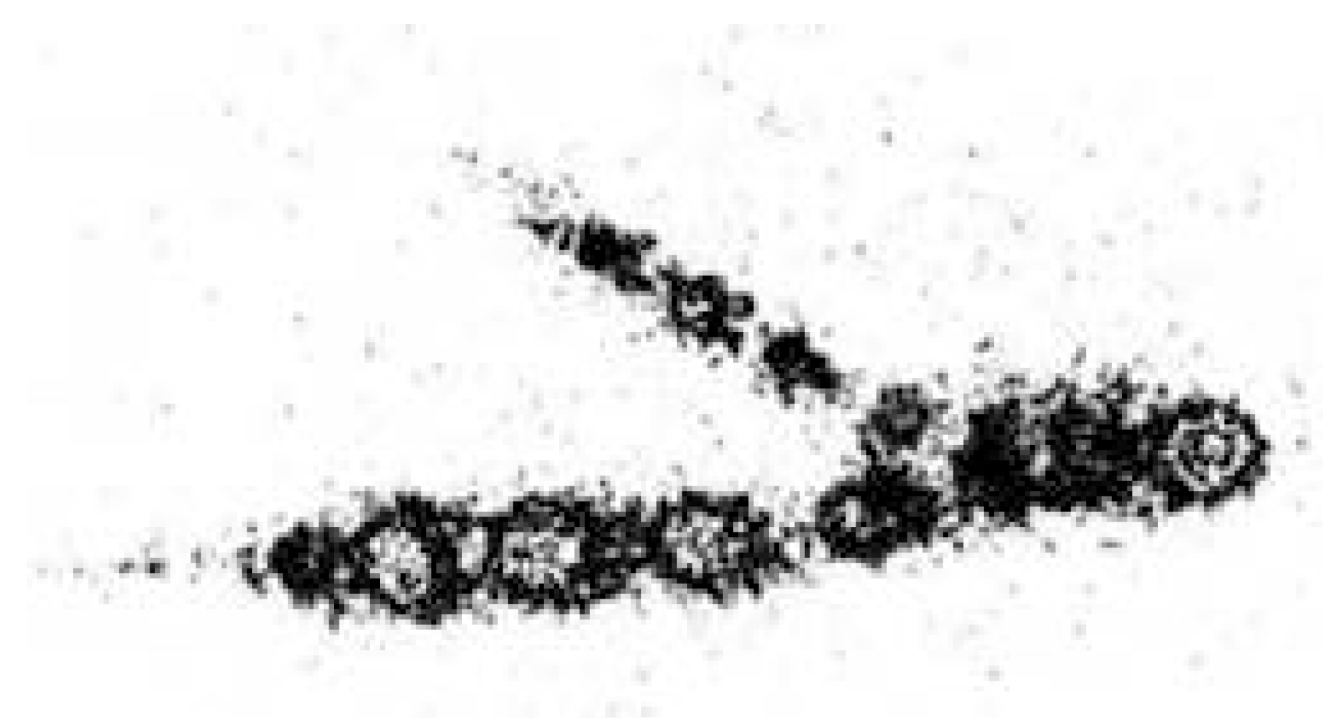

Figure 3. Expansion and compression zones in the atmosphere. 


\section{An Observation of Solar Electroscalar Radiation}

The most favourable conditions for the registration of longitudinal electroscalar waves are realized during a solar eclipse. During the eclipse, the Moon shields most of the flux of the transverse electromagnetic solar waves, while the longitudinal electroscalar waves having a greater penetration power do reach the Earth's surface. The incident solar radiation may lead to self-excited radial oscillations of the valence electrons in matter which are, in their turn, a source of electroscalar radiation. With this in view, metallic spheres were used to register such radiation. The result of measurements is displayed in Figure 4.

The horizontal axis shows the eclipse time with the start at 1p.m. on 1 August 2008. Along the vertical axis, the volt value of the detected signal is plotted. The results of measurements indicate the absence of transverse polarization of the incident solar radiation which passed through the Moon, the independence of the radiation amplitude from the position of the registration system outside or inside the copper box, as well as the large penetration ability of the electroscalar radiation (the thickness of the copper box walls was $5 \mathrm{~mm}$ ). The maximal signal was registered at 2.38 p.m. and amounted to 1.04 DC volt. The peak obtained in the measurements apparently attests that in this experiment one more signal was detected in addition to the electroscalar one. This can be explained by the following: in the case of a purely electroscalar signal one can expect a dip in the curve instead of a peak because the dip in the curve should depend on the depth of absorption of the electroscalar waves by the Moon. The most reasonable explanation for the registered peak is that scalar gravitational waves were detected together with the electroscalar waves. This assumption is grounded on the fact that the tidal gravitational forces from the Moon and the Sun during a solar eclipse are summed together, with the resulting force reaching its maximum. So, it is the unified electroscalar and scalar-gravitational field that was detected in this experiment. If the hypothesis for the existence of a unified field is confirmed, new perspectives will open up for the measurement of gravitational effects.

The above conclusion is substantiated by another example of using the unified electroscalar and scalargravitational interaction. The large amplification of the unified signal is expected during the regular motion of the Moon in its neomenia phase when the Moon is between the Earth and the Sun. Figure 5 shows the signal values versus the time of the New Moon.

The amplitude of the signal was 0.45 volt and its duration was 10 - 13 hours. The motion of any body equipped with an electroscalar field detector will produce an electric signal due to the combined fields of the Sun and the Moon.

The four copper spheres placed in a metallic screen were used to collect data on the solar radiation. The centers of the four spheres were connected to each other with a copper wire. The detector had a splitter for

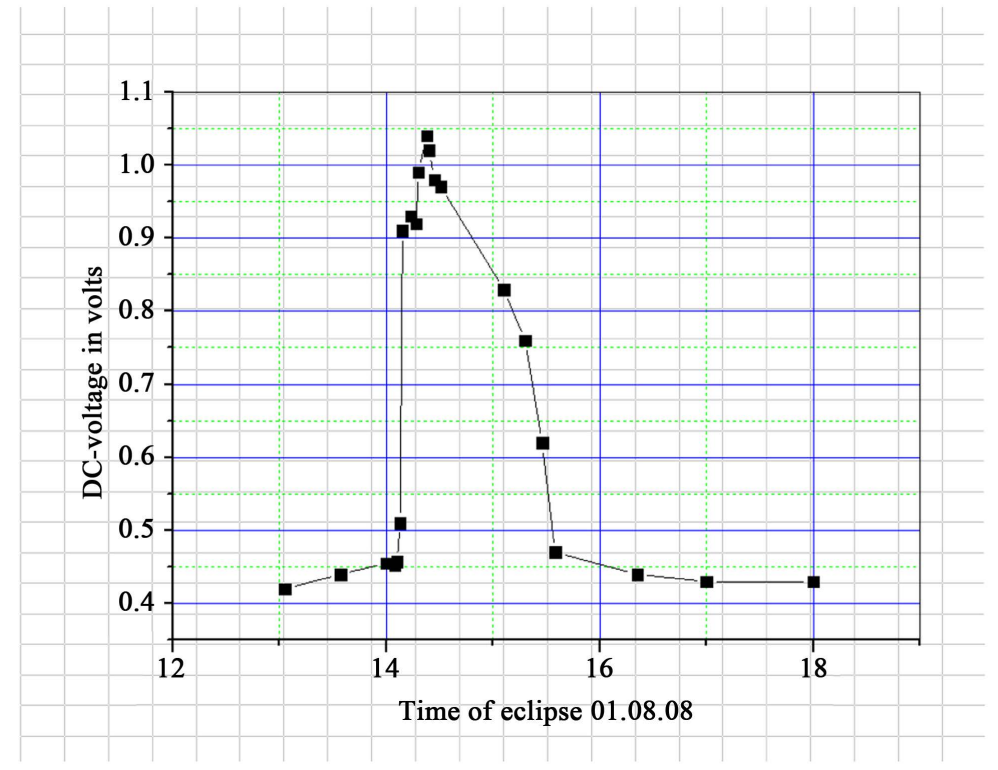

Figure 4. The detected signal from the sun. 


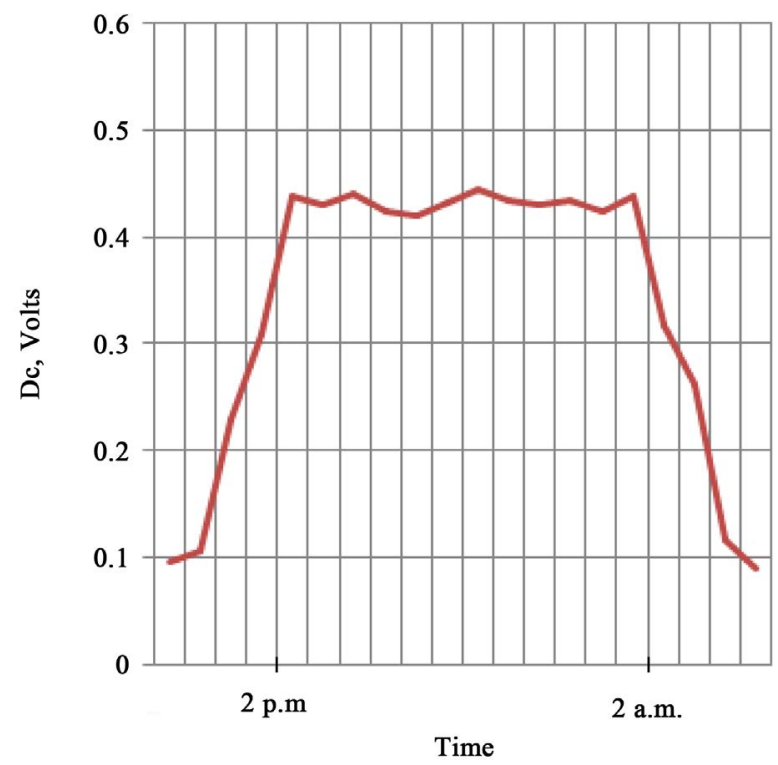

Figure 5. The unified signal during the New Moon.

signals and a system of amplitude-digital analyzers. This detector was able to register only electroscalar radiation. The electroscalar radiation is produced by the structures of solar plasma providing a spectrum having a discrete character with a sign-alternating amplitude of order of 20 millivolt and a frequency (by Fourier) up to 500 Hertz. The averaged value of the spectrum is negative and equals minus 2 - 5 millivolt, with a positiveamplitude signal coming on every millisecond and a negative-amplitude signal following after an interval. The frequency analysis of the spectrum shows that this radiation has a long wavelength and a practically constant amplitude. A single radiation signal has an exceedingly small value of raising time of electrical signal of front and drop of the order of fractions of a picosecond.

The spectrum of the solar electroscalar radiation is shown in Figure 6.

\section{Spectral Expansion of the Electroscalar Field}

The electroscalar field produced by charges may be expanded into a Fourier integral. If we present the electroscalar field as a superposition of plane waves, then the "frequency" of these waves will have a zero value and this field will not be time dependent while the wave vectors will be distinct from zero. The potential of this field is determined using the equation:

$$
\Delta \lambda=-4 \pi\left(\rho_{\|}+\frac{1}{4 \pi} \frac{1}{c} \rho_{0}\right) .
$$

We will now expand the electroscalar field potential $\lambda$ into the Fourier space integral:

$$
\lambda=\int_{-\infty}^{+\infty} \exp (i k r) \lambda_{k} \mathrm{~d}^{3} k
$$

We find next the Laplacian for the left-hand side:

$$
\Delta \lambda=-\int_{-\infty}^{+\infty} k^{2} \exp (i k r) \lambda_{k} \mathrm{~d}^{3} k
$$

and the Fourier k-component is: $\Delta \lambda_{k}=-k^{2} \lambda_{k}$. By defining the Fourier component from the right-hand side, we get:

$$
\Delta \lambda_{k}=-k^{2}\left(\frac{\rho_{\|}}{2 \pi^{2}}+\frac{\rho_{0}}{c 2 \pi^{3}}\right)
$$




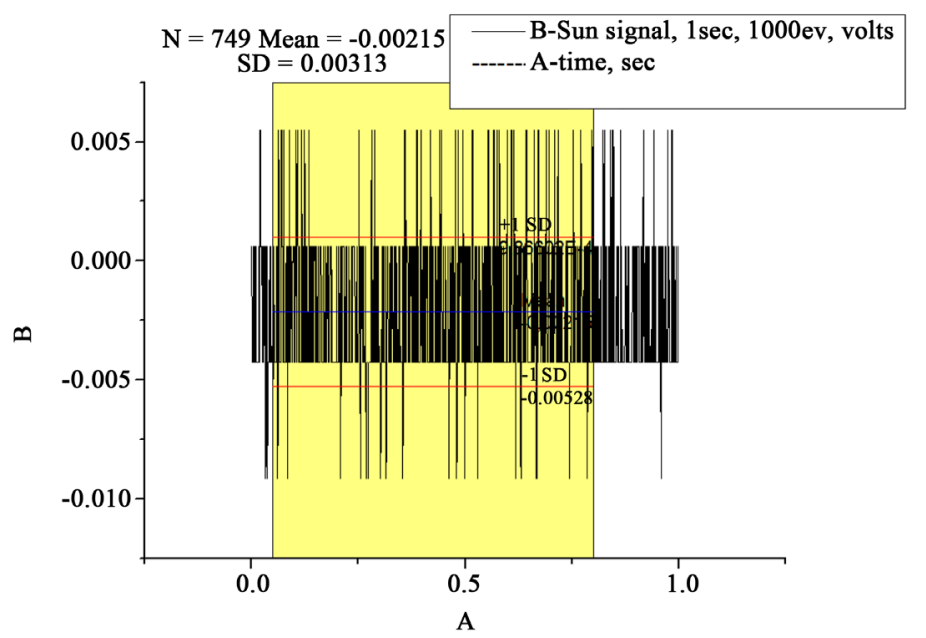

Figure 6. Spectrum of the solar electroscalar radiaton.

Then, we obtain by comparing both sides:

$$
\lambda_{\mathrm{k}}=\frac{\rho_{\|}}{2 \pi^{2}}+\frac{\rho_{0}}{2 \pi^{3} c} .
$$

Similarly to the field potential, the electroscalar field strength may be expanded as follows:

$$
\mathbf{E}_{\|}=\nabla \int_{-\infty}^{+\infty} \exp (i k r) \lambda_{k} \mathrm{~d}^{3} k
$$

We now insert $\lambda_{k}$ and have:

$$
\overrightarrow{\mathbf{E}}_{\|}=i \overrightarrow{\mathbf{k}}\left(\frac{\rho_{\|}}{2 \pi^{2}}+\frac{\rho_{0}}{2 \pi^{3} c}\right) .
$$

One may see from this expression that the electroscalar field is directed along the wave vector and its waves are longitudinal.

\section{The Degree of Freedom of a Charged Particle}

A peculiarity of the electrodynamics of electromagnetic and electroscalar fields is that the different characteristics are caused by changing in the behavior of a heavy charged particle. Due to this a strong correlation between the spatial position of the particle and the electric components of the fields can be possible [22]. It is known that the function of Lagrange remains invariant when the total derivative of any function is added [23]. Thus, we have: $L_{1}+L_{2}=0$ and $L_{2}=\frac{e}{c} \int \frac{\mathrm{d}}{\mathrm{d} t}[\lambda \mathrm{d} s]$, where $\mathrm{d} s=\sqrt{-\mathrm{d} x_{i}^{2}}$ and $i=1,2,3,4$.

So, we obtain for the action:

$$
\mathbf{S}=\left(-m c \mathrm{~d} s+\frac{e}{c} A_{i} \mathrm{~d} x_{i}\right)-\left.\frac{e}{c} \lambda \mathrm{d} s\right|_{a} ^{b}=0 .
$$

In the case when the upper limit is not equal zero, therefore the variation in action becomes:

$$
\delta \mathrm{S}=\delta \int\left(m c u_{i} \delta \mathrm{d} x_{i}+\frac{e}{c} A_{i} \delta \mathrm{d} x_{i}+\frac{e}{c} \delta A_{i} \mathrm{~d} x_{i}\right)-\frac{e}{c} \delta \lambda \mathrm{d} s-\frac{e}{c} \lambda \delta \mathrm{d} s=0 .
$$

Let us integrate the first, second and fifth terms by parts: finally we have following :

$$
\left.\left(m c u_{i}\left(1+\frac{e \lambda}{m c^{2}}\right)+\frac{e}{c} A_{i}\right)\right|_{a} ^{b}=0,
$$




$$
\frac{\mathrm{d} u_{i}}{\mathrm{~d} s}=-\frac{e}{C} \mathbf{Z}_{\mathbf{i k}} u_{k}+\frac{e}{c} \mathbf{F}_{\mathbf{i k}} u_{k} .
$$

where the equation for the particles' motion content the second-order tensor $Z_{i k}$ of the electroscalar field and the $F_{i k}$ electromagnetic tensor of second-order.

So, the electroscalar and electromagnetic tensors are:

$$
\begin{gathered}
\mathbf{Z}_{\mathbf{i k}}=\left(\frac{\partial \lambda}{\partial x_{i}} u_{k}-\frac{\partial \lambda}{\partial x_{k}} u_{i}\right) \\
\mathbf{F}_{\mathbf{i k}}=\left(\frac{\partial A_{i}}{\partial x_{k}}-\frac{\partial A_{k}}{\partial x_{i}}\right)
\end{gathered}
$$

Note that the electroscalar force has a negative sign with respect to the electromagnetic one. The Lagranges' method allows one to establish a strong correlation between the spatial position of the particle and the field components. The trajectories of propagation of the electromagnetic photon and electroscalar "scaphon" radiation may be different.This correlation directly points to a new degree of freedom in the electrodynamics of the charged particle, it providing uncontroversial proof that the electroscalar dynamics exists alongside with the electromagnetic one. It should be noted that in the course of transformations of the electromagnetic field into the electroscalar one and vice versa the particle may travel considerable distances and its trajectories may be spaced apart. The transformation of the electromagnetic field into the electroscalar one can also take place in the spheres and cavities of solid bodies that are different in type.

\section{Conclusions}

As follows from the theoretical and experimental studies, the electroscalar radiation has a long wavelength, is longitudinal and extremely penetrating. Basically, the following conclusions can be made:

1. The detection of the electroscalar radiation from the Sun was done using spherical electroreceptors in an eclipse of the Sun and during the regular motion of the Moon.

2. An analogy with the elasticity equation was made, and due to this the equation for the Maxwell electromagnetic field was derived from the transcendental component of the displacement vector, and an equation for the electroscalar field, from the longitudinal component of the displacement vector.

3. The longitudinal electroscalar wave performs transport of the Coulomb field, which is absent in the electromagnetic field.

4. The registered spectrum of the electroscalar radiation can be described by the law of propagation of the plane wave. The ratio of both components and their directionality are dependent on the type of medium. In vacuum both vectors are directed along the Pointing vector, while in the solid state the electric vector is directed against the motion, and the scalar one, along the wave motion.

5. The continuity equation of the electromagnetic field contains displacement of current, and the electroscalar field contains displacement of charge.

6. The spectral expansion of the electroscalar field testifies that the field is longitudinal since the electric vector is collinear to the vector of propagation.

7. The transfer of energy and information can take place either in vacuum or in any medium.

8. The degree of freedom of a massive charged particle due to the correlation between the space position of the particle and the field component gives the transformation of the electromagnetic field into the electroscalar field (and backwards) which occurs at the expense of the rotation of the transcendental electric vector round the magnetic field vector.

9. In the equation for the particle motion in the electroscalar field, the particle mass changes at the expense of the field energy, and the particle energy in the electroscalar field assumes a negative value to the sign of mechanical energy of motion.

10. An electrically charged particle displays dual properties which are revealed in its behavior as a wave and as a particle. The electromagnetic field of the particle shows wave properties during the interaction, and the electroscalar field acts as a particle due to the large value of the longitudinal force.

One remarkable characteristic of the electrodynamics of electroscalar and electromagnetic fields is the 
manifestation of different properties by a charged particle. This points to a possibility that the changes entailed by the new electroscalar dynamics are of profound nature and do not come into contradiction with those properties of electromagnetic dynamics which make it convincing and, consequently, such properties can be included into the electrodynamics as a second dynamics of charge motion.

\section{Acknowledgements}

I thank the Editor and the referee for their comments. I would like to thank Dr. D.V. Podgainy for his contribution and participation at the early stage of research. I am deeply obliged to Dr. Irina Molodtsova for assistanse in figure drawing.

\section{References}

[1] Zaimidoroga, O.A. and Podgainy, D.V. (2010) Observation of Electroscalar Radiation during an Eclipse of the Sun. Proceedings of International Conference of the Astroparticle and Cosmic Rays Physics, Como.

[2] Podgainy, D.V. and Zaimidoroga, O.A. Nonrelativistic Theory of Electroscalar Field and Maxwell Electrodynamics. arXiv:1005.3130.

[3] Podgainy, D.V. and Zaimidoroga, O.A. Relativistic Dynamics of a Charged Particle in an Electroscalar Field. arXiv:1203.2490.

[4] Dirac, P.A.M. (2002) Scientific Papers Collection. V. II. Quantum Theory (Scientific Articles 1924-1947). In: Sukhanov, A.D., Ed., M.: FIZMATLIT (Classics of Science).

[5] Fock, V. and Podolsky, B. (1932) Sowiet Physical Journal, 1, 801. Fock, V. (1964) The Theory of Space, Time and Gravitation. 2nd Revised Edition, Oxford, London, Paris.

[6] Polubarinov, I.V. (2003) Physics of Elementary Particles and Atomic Nuclei, 34, 738. Ogievezky, V.I. and Polubarinov, I.V. (1965) Journal of Nuclear Physics, 4, 216.

[7] van Vlaenderen, K.J. and Waser, A. (2001) Hadronic Journal, 24, 609.

[8] van Vlaenderen, K.J. A Generalisation of Classical Electrodynamics for the Prediction of Scalar Field Effects. arXiv:physics/0305098v1 [physics.class-ph].

[9] Khvorostenko, N.P. (1992) Russian Physics Journal, 35, 24-29 (Translated from Izvestiya Vysshikh Uchebnykh Zavedenii, Fizika, No. 3, 24-29, March 1992).

[10] Rousseaux, G. (2005) Europhysics Letters, 71, 15-20. http://dx.doi.org/10.1209/epl/i2005-10059-5

[11] Rousseaux, G., Kofman, R. and Minazzoli, O. (2008) The European Physical Journal D, 49, 249-256.

[12] Dirac, P.A.M. (1932) Proceedings of the Royal Society, A136, 453.

[13] Podolsky, B. and Fock, V. (1932) Sowiet Physical Journal, 2, 275-277.

[14] Bogoliubov, N.N. and Shirkov, D.V. (1984) Introduction to the Theory of Quantum Fields. Nauka, Moscow.

[15] Monstein, C. and Wesley, J.P. (2002) Europhysics Letters, 59, 514. http://dx.doi.org/10.1209/epl/i2002-00136-9

[16] Ignatiev, G.F. and Leus, V.A. (1999) On a Superluminal Transmission at the Phase Velocities. In: Chubykalo, A.E., Pope, V. and Smirnov-Rueda, R., Eds., Instantaneous Action at a Distance, Modern Physics: Pro and Contra, Nova Science Pub Inc., 203.

[17] Johansson, L. (1999) Longitudinal Electrodynamic Forces and Their Possible Technological Applications. Master of Science Thesis, CODEN:LUTEDX/(TEAT-5027)/1-55/(1996). http://www.df.lth.se/snorkelf/Longitudinal/Slutdok.html and Contra, p. 203 (1999)

[18] Love, A.E.H. (1927) A Treatise on the Mathematical Theory of Elasticity. Cambridge University Press, Cambridge.

[19] Landau, L.D. and Lifshitz, E.M. (1986) Theory of Elasticity, Vol. 7. 3rd Edition, Butterworth-Heinemann.

[20] Dubrovskii, V.A. (1985) An Elastic Model of the Physical Vacuum. Akademiia Nauk SSSR, Doklady, Vol. 282, No. 1, 83-88.

[21] Zalikhanov, B.Zh. (2016) Physics of Elementary Particles and Atomic Nuclei, 1, Dubna.

[22] Bell, J. (1966) Review of Modern Physics, 38, 447.

[23] Landau, L.D. and Lifshitz, E.M. (1987) Mechanics. Publishing Group, Nauka. 